\title{
Democracia y derechos de las personas LGBTI en América Latina: reformas para garantizar el derecho a la identidad y el derecho al voto de las personas trans, 2012-20201
}

\author{
Betilde Muñoz-Pogossian*
}

https://doi.org/10.35242/RDE_2020_30_4

Nota del Consejo Editorial

Recepción: 3 de junio de 2020.

Revisión, corrección y aprobación: 30 de junio de 2020.

Resumen: Un principio básico de las democracias es la igualdad, es decir, que todas las personas puedan gozar de los mismos derechos ante la ley (igualdad de jure), sin excepción alguna. Un reto mayor es traducir esa igualdad, que está en el papel, en la cotidianidad de las personas de forma que también en los hechos puedan gozarla; es esto a lo que debe aspirar un régimen democrático. Un grupo históricamente excluido, que no puede acceder a sus derechos en igualdad de condiciones son las personas LGBTI y en este grupo poblacional, las personas trans son generalmente sujetos de vulneraciones adicionales. Utilizando la Base de datos de reformas políticas (UNAM, 2020), el presente documento repasa las principales reformas ocurridas en las Américas para garantizar los derechos político-electorales de las personas trans, en particular, el derecho al reconocimiento de la identidad de género y el derecho al voto.

Palabras clave: Democracia / Derechos humanos / Derechos político / Derechos civiles y políticos / Igualdad de oportunidades / Población LGBTI / Derecho a la identidad / Derecho al sufragio / Garantías electorales / Reforma política.

Abstract: A basic principle of democracies is equality, that is, all people can enjoy the same rights before the law without any exception. An even greater challenge is to translate this equality, written in paper, into the daily lives of the people so that they can also enjoy it in real life; this is the aspiration of a democratic regime. A historically excluded group, which cannot have access to their rights in equal conditions, is the LGBTI people and, within this group, Trans people are generally subject to additional violations. This article, using the data bases of Political Reforms (UNAM, 2020), provides a review of the main electoral reforms that have taken place in the Americas to guarantee the political and electoral rights of Trans people, particularly the right to the acknowledgement of gender identity and the right to suffrage.

Key Words: Democracy / Human rights / Political rights / Civil and political rights / Equality of opportunities / LGBTI population / Right to identity / Right to suffrage / Electoral guarantees / Political reform.

\footnotetext{
${ }^{1}$ Investigación realizada gracias al Programa de Apoyo a Proyectos de Investigación e Innovación Tecnológica (PAPIIT) de la UNAM. Clave del proyecto: IN103020. La autora agradece a Tamara Adrián y Juan Pablo Delgado, por las revisiones a versiones previas de este manuscrito.

*Venezolana-estadounidense, politóloga, correos bvmr@hotmail.com y bmunoz@oas.org. Tiene 20 años de trayectoria en el ámbito internacional. Doctora en Ciencias Políticas de la Florida International University en Miami, Florida (USA) y tiene una maestría en Relaciones Internacionales de la University of South Florida en Tampa, Florida (USA). En la OEA, lidera el trabajo en derechos económicos, sociales y culturales, la inclusión social de grupos en situaciones vulnerables como los afrodescendientes, indígenas y personas con discapacidad, y la promoción de los derechos humanos y la inclusión social de migrantes y refugiados. Sus más recientes publicaciones incluyen el libro Women, Politics and Democracy (2017) publicado por Palgrave McMillan y el artículo "Where are the Women? Why Expanding our Understanding of Venezuela's Humanitarian Crisis Matters"? publicado por el Georgetown Journal of International Affairs, mayo 11, 2020.
} 


\section{DERECHO ELECTORAL}

\section{INTRODUCCIÓN}

La democracia es una forma de organizar el poder en la sociedad, que busca ampliar el ejercicio de los derechos humanos de todas las personas, evitar o limitar la dominación de personas o grupos que impidan ese objetivo al tiempo que debe lograr la perdurabilidad de la organización democrática (Munck, 2005 y 2009; Munck y Verkuilen, 2002 y PNUD, 2010 p. 33). Un principio básico de las democracias es la igualdad, es decir, que todas las personas puedan gozar de los mismos derechos ante la ley (igualdad de jure), sin excepción alguna. Un reto mayor es traducir esa igualdad, que está en el papel, en la cotidianidad de las personas de forma que también en los hechos puedan gozarla; es esto a lo que debe aspirar un régimen democrático.

En efecto, la democracia debe buscar ampliar la ciudadanía integral, lo que implica avanzar hacia el pleno reconocimiento de la ciudadanía de todas las personas en sus tres dimensiones: política, civil y social (PNUD, 2010, p. 31 y 2004, p. 24). Bajo este marco, todas las personas, sin discriminación alguna, deberían poder insertarse en la sociedad y gozar, en las leyes y en las prácticas, de todos sus derechos civiles, sociales, políticos, económicos, culturales y ambientales, ya que todos estos derechos comprenden un conjunto interdependiente, indivisible $y$ articulado.

En la región; sin embargo, se constatan y replican, permanentemente, asimetrías y desigualdades en el acceso, goce y pleno ejercicio de los derechos de muchos ciudadanos y ciudadanas. En el ámbito público, y por diversas razones, los derechos político-electorales han sido y siguen siendo objeto de limitaciones en sus dos facetas, el derecho a elegir y a ser electo, para muchas personas. A lo largo de la historia, se ha estudiado ampliamente las vulneraciones a los derechos políticoelectorales de las mujeres por estar insertas en una democracia androcéntrica que las ha excluido del ejercicio del poder y de la participación política.

Sin embargo, las personas lesbianas, gays, bisexuales, trans e intersex (LGBTI), y en específico las personas trans, han sufrido particulares vulneraciones, discriminaciones y violencias por medio de "la anulación de su dignidad como persona[s], y por ende, su consecuente invisibilización y negación de acceso a lo público" (Garza, 2019, pp. 18-19); acciones totalmente incompatibles con un Estado de derecho democrático, y que 


\section{DERECHO EIECTORAL}

atentan contra sus principales postulados. Las exclusiones y violencias que experimentan estas personas se complejizan, también, por los efectos de la interacción de diferentes factores de discriminación adicionales a su condición de diversidad sexual tales como su origen social, identidad étnica y/o lingüística, edad, diversidad funcional, condición de salud, condición migratoria, entre otros (INE, 2018, p. 12).

Es importante reconocer; sin embargo, que la región lleva varios años implementando reformas para acercar derechos a las poblaciones LGBTI. Por ejemplo, quince de veinte países (Argentina, Bolivia, Brasil, Chile, Colombia, Costa Rica, Cuba, Ecuador, El Salvador, Guatemala, México, Nicaragua, Perú, Uruguay y Venezuela) tienen algún tipo de protección o leyes que protegen a esta ciudadanía contra la violencia y/promueven los derechos de su ciudadanía LGBTI.

Países como como Argentina, Colombia, Costa Rica, Brasil, Ecuador, México y Uruguay reconocen el matrimonio entre personas del mismo sexo a nivel nacional. En Panamá y Chile, se están teniendo debates nacionales sobre el tema. Igualmente, catorce países en América Latina han prohibido explícitamente la discriminación en el lugar de trabajo debido a la orientación sexual. Si bien los países de las Américas se encuentran en diferentes etapas de avance en cuanto al acceso a derechos de estas poblaciones, se considera que el hemisferio está más avanzado si lo comparamos con otras regiones del mundo como Medio Oriente, África y Asia $^{2}$. Por ejemplo, a nivel global, 83 países continúan penalizando las relaciones sexuales entre personas del mismo sexo. En América Latina, esto ya no es penalizado ${ }^{3}$ y en años recientes, se ha realizado un avance significativo a nivel legislativo para garantizar los derechos humanos de las personas LGBTI.

En materia político-electoral; sin embargo, mientras que van ya muchos años de reformas orientadas a facilitar el ejercicio de los derechos políticoelectorales de las mujeres (Freidenberg y Muñoz, 2016), son recientes y escasas las reformas en América Latina que han estado orientadas a acercar derechos político-electorales a las poblaciones LGBTI y a las personas trans particularmente. Es trascendental analizar esto en clave democrática, ya que, a falta de reconocimiento legal de la identidad, las

\footnotetext{
${ }^{2}$ Para más información comparativa en el avance de los derechos de las poblaciones LGBTI en el mundo, ver http://www.equaldex.com/

${ }^{3}$ Con una excepción, Venezuela en el ámbito militar bajo el Código de Justicia Militar.
} 


\section{DERECHO ELECTORAL}

personas trans se enfrentan a obstáculos casi insuperables para ejercer la participación política en las dos facetas de estos derechos (elegir y ser electas), y en los países en los que ya existe ese reconocimiento, probablemente habría que pensar en reformas que incorporen cuotas para aumentar la posibilidad de representación de estas poblaciones. De cualquier modo, en la región las reformas avanzadas han estado limitadas al derecho al voto, a través de reformas en materia de identidad de género y facilitando así el derecho a votar. Al mismo tiempo, y desde una perspectiva de aprendizaje en materia institucional, las reformas políticoelectorales en la región que se enfocaron, primero, en conceder condiciones plenas en el ejercicio del voto a las mujeres, y mucho más adelante, en incrementar su representación en el ámbito político (para ejercer el poder) han marcado una pauta sobre las medidas que los Estados pueden tomar para dejar de tener democracias incompletas y transitar hacia democracias más efectivas e inclusivas, y estas pueden ser útiles a la hora de diseñar reformas futuras para proteger los derechos político-electorales de las personas LGBTI. Para el caso particular de las personas trans, aunque es vital revertir las violencias y discriminaciones que tienen lugar en todas las facetas de su ciudadanía, las escasas, pero decididas reformas recientes se han enfocado en su derecho a existir y ser visibilizadas como personas (derecho a la identidad) y de ser sujetos políticos (derecho a votar), entre otras.

Usando datos provenientes del Observatorio de Reformas Políticas en América Latina de la Universidad Autónoma de México (UNAM) y la Organización de los Estados Americanos (OEA) ; este artículo busca mapear las principales reformas llevadas a cabo en la región respecto de los derechos político-electorales de las personas trans como personas que requieren protección y de acciones afirmativas deliberadas para revertir su situación de exclusión política. La primera sección se ocupa de revisar la literatura en la materia, para evidenciar cuáles son las principales miradas que le han dado las ciencias políticas a este tema, así como el aporte que hace el presente estudio. Por su parte, la segunda sección está dedicada a revisar el marco jurídico internacional que ha buscado la protección de derechos de las personas LGBTI y, en particular, de las personas trans. Por ser un área poco investigada por la ciencia política, esta temática tiende a provocar confusión de términos y conceptos; es por esto, que en la tercera sección se abordan los principales conceptos y se

\footnotetext{
${ }^{4}$ Para más información sobre el Observatorio, ver https://reformaspoliticas.org/
} 


\section{DERECHO ELECTORAL}

operacionalizan las definiciones sobre las que descansará el análisis. Mientras que la cuarta parte ahonda sobre la naturaleza, los caminos, y los alcances de las principales reformas en América Latina respecto al derecho a la identidad y al voto de las personas trans. La última sección plantea la agenda pendiente en materia de reformas político-electorales para las personas LGBTI y, en particular, las personas trans.

\section{Derechos LGBTI: Miradas desde la ACADemia}

Una revisión de la literatura académica en las ciencias políticas revela la poca existencia de estudios enfocados en los procesos políticos de reforma para la protección de los derechos político-electorales de las personas LGBTI. Aunque en la sociología, la antropología, la historia y los estudios culturales, se aborda la temática LGBTI como parte central del estudio teórico, se han publicado pocos estudios sobre temas LGBTI en revistas de ciencias políticas en los Estados Unidos (Corrales y Pecheny, 2010), y mucho menos en revistas latinoamericanas. La falta de atención a esta temática no se corresponde con el constante interés de las politólogas en entender procesos de democratización y de reformas del Estado para proteger derechos, de las cuales la política LGBTI es sin duda central. Es difícil entender las razones de esta escasa producción académica, aunque pueden estarse replicando los patrones de desigualdad y exclusión que existen en la sociedad a la hora de pensar las preguntas e hipótesis en el campo de estas ciencias.

En años recientes, esto ha comenzado a cambiar, y aunque se encuentran pocos abordajes rigurosos que den cuenta de las limitaciones en el ejercicio de derechos político-electorales de estas personas, o de las reformas avanzadas y las pendientes, la serie de estudios que sí están disponibles pueden agruparse en (i) aquellos que se ocupan de los factores internos o de empuje que promueven las reformas y que, entre otras cosas, evalúan la potencia y efectividad de los movimientos sociales y organizaciones en el terreno. Enfocándose también en factores internos, (iii) un grupo de autores ha estudiado el rol de las ideologías en facilitar o no la aprobación de reformas en la agenda LGBTI.

Acorde con el tiempo político que vive la región, (ii) una reciente área de investigación asociada a este marco de análisis está empezando a investigar las resistencias a este mayor goce de derechos de las personas LGBTI o backlash, con énfasis particular en la religión como eje central de 


\section{DERECHO ELECTORAL}

las resistencias. Finalmente, se encuentran (iv) aquellos que evalúan los factores externos, incluyendo a los que desde una mirada más internacionalista estudian el efecto difusión (difusion effect), o el efecto que los avances y progresos en el goce de derechos LGBTI en un lugar (usualmente democracias consolidadas) tienen en los países de la región.

Gran parte de los estudios analizan los factores internos o de empuje (push factors) y los externos (pull factors) que independiente o conjuntamente producen reformas legales por más derechos para las personas LGBTI. Entre quienes estudian los factores internos, de la Dehesa (2020) analiza los casos de las dos democracias más grandes de América Latina, México y Brasil, centrándose en factores internos de empuje por reformas por más derechos tales como las formas en las que activistas LGBTI se han involucrado con el Estado, particularmente en alianza con los partidos políticos, y a través de las agencias de salud del gobierno, y evaluando el compromiso de los Estados en el contexto más amplio de las transiciones políticas hacia la democracia, la transformación neoliberal de las relaciones entre el Estado y la sociedad civil y la consolidación gradual de los derechos sexuales a nivel internacional. En este sentido, también toca el ámbito relacionado con el cuerpo de literatura que aborda el Ilamado "efecto difusión".

Una serie de académicos se han abocado a analizar el rol de las ideologías de los gobiernos de turno al momento de las reformas (Friedman y Tabbush, 2019; Tabbush et al., 2019; Elfenbein, 2019 y Larracoechea, 2019). Estos estudios evalúan cómo la izquierda o "Marea Rosa" que lideró muchos de los gobiernos nacionales en la región en un momento reciente de la historia latinoamericana abordó los derechos basados en el género y la diversidad sexual, y hacen la pregunta, con respuestas bastante variadas de país a país, sobre si estos gobiernos de izquierda fueron un factor para motivar y promover reformas por los derechos LGBTI. Al tiempo que estos estudios reconocen que muchos de estos gobiernos, por ejemplo, lograron la legalización del matrimonio igualitario o aprobaron reformas para el reconocimiento de la identidad de género, también operaron en relaciones de poder heteropatriarcales, ignorando o rechazando los elementos más desafiantes de una agenda social y participando en intercambios estratégicos entre género y derechos sexuales.

Por su parte, Corrales y Sagarzazu (2019) señalan que la literatura entre los latinoamericanistas ha sido enormemente fructífera en la producción 


\section{DERECHO EIECTORAL}

de ideas teóricas sobre las condiciones bajo las cuales los grupos de presión pueden empoderarse a nivel local ${ }^{5}$. Sin embargo, estos autores ponen la atención en los factores de resistencia, a saber, grupos de interés, y actores de influencia que resisten los progresos en el alcance de estos derechos y operan para revertir esos avances.

En su análisis, y usando el caso de Colombia, Corrales y Sagarzazu (2019) señalan que la mayoría de los estudios se han centrado en el lado de los factores de empuje, es decir, las circunstancias que llevan a los actores aparentemente débiles y marginados a superar la resistencia y cambiar las políticas y leyes estatales, pero indican que es clave estudiar las resistencias, especialmente, y tal como demuestran, el rol de religión en la propagación de actitudes homofóbicas y transfóbicas, con la advertencia de que no todas las religiones, y quienes las practican las manifiestan con la misma fuerza (Corrales y Sagarzazu, 2019). En estudios recientes, Corrales (2020) señala también a algunas iglesias evangélicas como el actor que más resistencia está dando a estos avances, y el que mejor ha sabido usar las instituciones democráticas para bloquear nuevos progresos en esta agenda.

Por último, están los estudios que se han dedicado a analizar factores externos, en particular el "efecto difusión" (Friedman, 2000; Encarnación 2016; Corrales y Pechney, 2010 y Corrales y Sagarzazu, 2020). Mientras que Friedman (2012), Corrales y Pechney (2010), entre otros, argumentan que países como España y otros, y sus progresos en materia de derechos LGBTI han sido la fuerza externa impulsora del avance de estos derechos en América Latina (Friedman, 2012); otros como Encarnación (2016) y Diez (2016) toman distancia de las explicaciones puramente internacionalistas y evalúan cómo lo local y lo global interactuan para hacer a la región uno de los entornos más receptivos del mundo para los derechos LGBTI, poniendo énfasis en el importante rol de activistas locales. Estas explicaciones dan mucho valor a movimientos nacionales que han visibilizado los reclamos de las personas LGBTI en sus países para plantear demandas que tienen receptividad a nivel nacional.

\footnotetext{
${ }^{5}$ Los autores señalan estos factores: la capacidad de estos grupos locales de (1) formar alianzas con otros movimientos sociales más grandes; 2) trabajar junto con partidos políticos compatibles; 3 ) colaborar con diferentes partes de la burocracia (es decir, el Estado) para diseñar programas, políticas o regulaciones; 4) usar el discurso para enmarcar su causa de manera que resuene con el público en general; 5) aprovechar la competencia de partidos o aperturas liberales en la legislatura, los tribunales y los gobiernos subnacionales, 6) elegir abiertamente representantes LGBT; 7) cultivar lazos con actores y tendencias transnacionales y 8) hacer un uso inteligente de las redes sociales.
} 


\section{DERECHO EIECTORAL}

Tal como muestra esta revisión bibliográfica, aunque ha habido un grupo importante de estudios que buscan explicar el éxito en los avances de las reformas por más derechos para las personas LGBTI, en general, pocos estudios politológicos se han enfocado en estudiar la naturaleza de las reformas, y la agenda pendiente en materia de derechos políticoelectorales de las personas LGBTI desde una concepción de ciudadanía integral y que puede tener efectos en cómo las personas trans pueden insertarse y ser visibilizadas en una sociedad democrática. La literatura en materia de democracia y reformas políticas desde América Latina; sin embargo, está empezando a estudiar cada vez más rigurosamente este tema. Aunque es insuficiente, en la literatura latinoamericana autoras como López (2019), Esparza (2019), entre otras, también se han abocado al tema de expansión de derechos $y$, en particular, del derecho a la identidad y al voto para las personas trans.

Vale resaltar la sistematización de datos en el tema llevada a cabo por el Observatorio de Reformas Políticas en América Latina de la UNAM-OEA, al igual que el avance en esta materia realizado por la Universidad de Guanajuato la cual está creando un Índice de Reconocimiento de Derechos LGBTI en América Latina ${ }^{6}$ para observar el comportamiento de los países en este ámbito, con particular atención a los que ya han dado pasos (Argentina, Uruguay, Colombia, Ecuador y México) evaluando los derechos que reconocen y el ritmo con el que han aparecido (López, 2019).

\section{MARCo Internacional por los DeRechos LGBTI: BReVe MiRADA a los derechos de las Personas Trans}

La Declaración Universal de los Derechos Humanos aprobada en el seno de las Naciones Unidas hace más de 60 años establece que "Todos los seres humanos nacen libres e iguales en dignidad y derechos". Este primer enunciado de la Declaración es el principio básico a nivel internacional para que las personas LGBTI puedan acceder plenamente a sus derechos en igualdad de condiciones que todos los otros seres humanos. Sin embargo, esto no ha ocurrido plenamente, y subsecuentes

\footnotetext{
${ }^{6}$ Este índice considera 15 indicadores, por ser el número de derechos aprobados en los cinco países que han aprobado reformas hasta ahora (Argentina, Uruguay, Colombia, Ecuador y México): derecho a la familia reproducción asistida-, matrimonio igualitario, uniones civiles, adopción, heredar a la pareja una vez que esta fallece, identidad de género, ejercicio de voto para las personas trans, salud -con una atención especial para las personas trans-, trabajo - con una atención especial para las personas trans-, beneficios sociales, intimidad privacidad de datos-, educación, pensión a la pareja, derecho a la vida -despenalización de la homosexualidady libertad de expresión.
} 


\section{DERECHO EIECTORAL}

instrumentos, vinculantes y no vinculantes, se han venido ocupando de tratar de revertir esta situación de desigualdad. Entre ellos, vale mencionar tres que son centrales en la agenda de derechos LGBTI, con miradas particulares a las personas trans: la Convención Interamericana Contra Toda Forma de Discriminación e Intolerancia (2013), los Principios de Yogyakarta sobre la Aplicación de la Legislación Internacional de Derechos Humanos en Relación con la Orientación Sexual y la Identidad de Género (2006), y finalmente, la Opinión Consultiva n. ${ }^{\circ} 24$ de la Corte Interamericana de Derechos Humanos (2019).

En lo que respecta a la región de las Américas, el debate político y consensos a nivel regional se fueron construyendo sobre la base de una serie de resoluciones en el marco de la Organización de los Estados Americanos (OEA) ${ }^{7}$ a partir de 2008, los cuales se enfocaron en abogar abiertamente por la lucha contra la violencia y violaciones de derechos humanos cometidas por motivos de orientación sexual e identidad de género, por condenar la discriminación y por alentar a los Estados miembros de la Organización para adoptar políticas públicas para proteger a esta población. Sin embargo, un hito importante de nuestra región, que de algún modo evidenció la maduración del debate político sobre la agenda de derechos LGBTI, fue la aprobación, en 2013, del primer instrumento jurídico interamericano y el único a nivel global que incluye a las personas LGBTI como sujetos de protección internacional: la Convención Interamericana Contra Toda Forma de Discriminación e Intolerancia ${ }^{8}$. La Convención reconoce que los Estados ${ }^{9}$ miembros de la OEA tienen "la obligación de adoptar medidas en el ámbito nacional y regional... sin distinción alguna por motivos de sexo, edad, orientación sexual, identidad y expresión de género...", entre otras categorías.

El contenido de la Convención es enfático, toda vez que habla de "erradicación total e incondicional" de toda forma de discriminación (OEA, 2013); es realista, ya que expresa su "alarma por el aumento de los crímenes de odio cometidos por motivos de sexo, religión, orientación

\footnotetext{
7 Para más información, ver las resoluciones de la Organización de Estados Americanos (OEA): AG/Res. 2435 (XXXVIII-O/08), AG/Res. 2504 (XXXIX-O/09), AG/Res. 2600 (XL-O/10), AG/Res. 2653 (XLI-O/11), AG/Res. 2721 (XLII-O/12), AG/Res. 2807 (XLIII-O/13) y AG/Res. 2863 (XLIV-O/14).

${ }^{8} \mathrm{Al}$ mismo tiempo que se aprobó esta Convención, se aprobó la Convención Interamericana Contra el Racismo, la Discriminación Racial y Formas Conexas de Intolerancia debido a que algunos países no querían suscribir un documento que tuviera a las personas LGBTI como sujetos de protección internacional.

${ }^{9}$ Hasta la fecha, esta Convención ha sido firmada por Argentina, Bolivia, Brasil, Chile, Colombia, Costa Rica, Ecuador, Haití, México, Panamá, Perú y Uruguay. Tanto México como Uruguay ya la han ratificado, por lo que la Convención ya entró en vigor.
} 


\section{DERECHO ELECTORAL}

sexual, deficiencia y otras condiciones sociales" (OEA, 2013); y es universal, porque establece que "todo ser humano es igual ante la ley y tiene derecho a igual protección contra toda forma de discriminación e intolerancia en cualquier ámbito de la vida pública o privada" (OEA, 2013).

En lo que respecta a derechos político-electorales, los Principios de Yogyakarta sobre la Aplicación de la Legislación Internacional de Derechos Humanos en Relación con la Orientación Sexual y la Identidad de Género de 2006 dan un paso más ratificando los estándares internacionales vinculantes para la protección de los derechos LGBTI y, además, dando una serie de orientaciones sobre cómo aplicar la legislación internacional de derechos humanos a las cuestiones de orientación sexual e identidad de género a nivel nacional. El Principio 25 se ocupa de la ciudadanía política al establecer que las personas de todas las orientaciones sexuales e identidades de género pueden participar en la vida pública en calidad de ciudadanas y ciudadanos, tanto en la conducción de los asuntos públicos, incluido el derecho a votar y postularse a cargos electivos, la incidencia en la formulación de políticas que afecten su bienestar, como el acceso, en condiciones de igualdad, a todos los niveles y empleo en funciones públicas (Principios, 2017). En lo referente a las personas trans, el Principio 2 advierte del impacto negativo de la discriminación para la realización de los derechos de las personas trans y la obligación de los Estados de contrarrestarla de manera amplia (Principios, 2017). En el Principio 3 se desarrolla el derecho al reconocimiento de la personalidad jurídica y la obligación del Estado de reconocer legalmente la identidad de género, lo que significa que a la persona se le deben proporcionar los medios para rectificar su documentación oficial y los datos registrados al momento de nacer, sin prerrequisitos médicos ni restricciones judiciales o de otro tipo (Principios, 2017). Se ocupa también de la protección de la privacidad de las personas trans. En su Principio 6, protege el derecho de las personas trans de elegir cómo, cuándo y a quién revelar información relacionada con su orientación sexual o identidad de género (Principios, 2017).

Aunque tanto la Convención mencionada como los Principios de Yogyakarta son claves para el avance de la agenda de derechos LGBTI, es la Opinión Consultiva n. ${ }^{\circ} 24$ de 2017 (OC n. ${ }^{\circ}$ 24/2017) de la Corte Interamericana de Derechos Humanos (Corte $\mathrm{DDHH}$ ) la que inicia un nuevo momento para el ejercicio de derechos para esta población, y se convierte en la visión de derechos LGBTI más progresista entre el corpus 


\section{DERECHO EIECTORAL}

iuris vigente del derecho internacional de los derechos humanos (PUICA, 2020a). Esta resultó de una solicitud del Estado costarricense para que la Corte emitiera su opinión sobre: (a) si tenía la obligación de extender los derechos de propiedad a las parejas del mismo sexo y (b) si debía permitir que las personas transgénero cambiasen su nombre en sus documentos de identidad.

En esta opinión, la Corte IDH determinó que el derecho de cada persona a definir su identidad de género de manera autónoma, así como el derecho a que la información personal que aparezca en sus registros y documentos de identificación correspondan con su identidad de género autopercibida son derechos protegidos por la Convención Americana de Derechos Humanos ${ }^{10}$. Otra importante contribución de la OC-24/17 de la Corte IDH es haber establecido estándares comunes para garantizar el reconocimiento pleno de las identidades de género no normativas en la región (PUICA, 2020a, p. 31).

\section{Diversidad Sexual: Conceptos Clave}

Para propósitos de este estudio, la palabra "sexo" se entiende como una categoría binaria que tiene su origen en una interpretación cultural e histórica que se ha hecho de las diferencias anatómicas y fisiológicas entre las personas, primordialmente asociadas con su genitalidad. Así, las personas "intersex", quienes presentan una o más variaciones respecto del modelo de corporalidad femenina o masculina, quedan fuera de esta tradicional clasificación. Quedan igualmente excluidas de esa concepción binaria hombre-mujer algunas identidades sexo-genéricas intersectadas con la identidad étnica como son las identidades muxe o biza'ah en México (Garza, 2020, p. 21).

\footnotetext{
10 Para dotar de verdadera efectividad los contenidos de la Opinión, la propia Corte propuso los siguientes procedimientos para reconocer la identidad de género: (a) naturaleza administrativa: es decir, la necesidad de implementar procedimientos de naturaleza formal y materialmente administrativa; (b) Integralidad: es decir, que se permita la rectificación de las menciones nombre y sexo/género en un solo acto administrativo, y que esto sea en todos los documentos y registros de las personas, y cuando corresponda, la captura de la fotografía acorde a la expresión de género de quien lo solicita; (c) Consentimiento libre e informado como único requisito para su tramitación, respetando el estándar convencional de respetar la identidad autopercibida de la persona, sin necesidad de pruebas médicas, psiquiátricas o de cualquier índole, que puedan resultar invasivas $y$ patologizantes; (d) Expeditez y gratuidad; (e) Confidencialidad; (d) Provisiones sobre niñez y adolescencia, indicando que las premisas anteriores son aplicables en su totalidad para las infancias y adolescencias por ser titulares del mismo derecho que las personas adultas a ser reconocidas en su identidad de género autopercibida (PUICA, 2020a, pp. 20-21).
} 


\section{DERECHO EIECTORAL}

Este sistema binario de clasificación entre mujeres y varones a veces no se corresponde con determinadas realidades, e impone una clasificación alineada con una construcción social de roles y estereotipos: expectativas sobre cómo relacionarse con otras personas que pertenezcan o no a un mismo sexo, cómo vestirse o cómo comportarse. A esta construcción social de roles y expectativas es a lo que llamamos género. En este marco, la identidad de género es el término utilizado para describir el sentir de las personas transgénero, las cuales se identifican con el género opuesto (CJI, 2013 y López, 2020). Es decir, que una persona puede sentir profunda e individualmente una identidad de género distinta al sexo que le fue asignado al momento de nacer. Por otro lado, la expresión de género se refiere a la manifestación externa de los rasgos culturales que permiten identificar a una persona como masculina o femenina conforme a los patrones considerados propios de cada género, por una determinada sociedad en un momento histórico específico (CJI, 2013 y López, 2020).

Dos conceptos que es vital también tener en cuenta a partir de las concepciones de identidad de género son el de ser una persona cisgénero y el ser una persona transgénero. Una persona cisgénero es aquella que se identifica con el sexo asignado al momento de nacer ${ }^{11}$, mientras que las personas transgénero o trans son aquellas que no se identifican con dicha asignación ${ }^{12}$ (PUICA, 2020a, p. 12). En el caso de las personas cuya identidad de género no se corresponde con el sexo al nacer, se les suele denominar personas transexuales, transgénero y travesti, con características especificas para cada una. Así:

- Transgénero es un término utilizado para aquellas personas que tienen identidad de género y expresión de género diferentes a su sexo biológico.

- Transexual, persona cuya identidad de género es diferente a su sexo biológico, estas personas por regla general modifican sus cuerpos para que su sexo coincida con su identidad de género.

\footnotetext{
${ }^{11}$ Etimológicamente el prefijo "cis" significa "de este lado" o "de aquí". Las personas cisgénero también son conocidas como personas con identidades de género normativas, en el entendido de que su identidad de género autopercibida está conforme a la norma socialmente aceptada denominada "cisnormatividad" (PUICA, 2020a, p. 12).

12 Etimológicamente el prefijo "trans" significa "a través", "más allá" o "de un lado al otro". Las personas trans también son conocidas como personas con identidades de género no normativas, en el entendido de que su identidad de género autopercibida no está conforme a la norma socialmente aceptada denominada "cisnormatividad" (PUICA, 2020a, p. 12).
} 


\section{DERECHO ELECTORAL}

- Travesti es la persona que se viste con ropa del sexo opuesto, pero que no necesariamente se identifica con ese género (CIJ, 2013).

Por un lado, un hombre trans puede ser identificado como una persona a la que se le ha asignado un sexo/género femenino al nacer, pero que rechaza esa asignación y se identifica dentro del espectro masculino a partir de la discursividad, dentro de un contexto histórico y social determinados. Este hombre trans puede asumirse heterosexual, homosexual o bisexual, por ejemplo (Garza, 2020, p. 20). Por el otro, una mujer trans, por el contrario, es una persona a quien se le ha asignado el sexo/género masculino al nacer, pero que se identifica como mujer en relación con su contexto social e histórico. La mujer trans también puede asumirse heterosexual, lesbiana o bisexual (Garza, 2020, p. 20). Para propósitos de esta investigación, se respetarán estas variaciones en la identificación con el eje común de considerar a los grupos de personas que encuentran una discrepancia entre el género asignado y el género construido. De forma que al utilizar el término "trans", se hablará de personas que se vean reflejadas en los tres términos arriba mencionados.

Por su parte, el término "orientación sexual" se enfoca en la atracción física o emocional hacia personas del mismo sexo, del sexo opuesto o de ambos sexos. Las personas "homosexuales" son aquellas que se sienten atraídas física y emocionalmente por personas de su mismo sexo; por su parte, a quienes sienten atracción por personas de distinto sexo se les denomina "heterosexuales". Las personas "bisexuales" son aquellas que se sienten atraídas por personas de su mismo sexo y también del sexo opuesto $y$, finalmente, a quienes no se sienten atraídas sexualmente hacia otras personas se las denomina asexuales. Se habla de un sesgo cultural a favor de las relaciones heterosexuales, "las cuales son consideradas normales, naturales e ideales y son preferidas por sobre relaciones del mismo sexo o del mismo género. Ese concepto apela a reglas jurídicas, religiosas, sociales y culturales que obligan a las personas a actuar conforme a patrones heterosexuales dominantes e imperantes" (PUICA, 2020b, p. 5).

El rango de orientación sexual, identidad y expresión de género es tan amplio y diverso como la diversidad humana. Al hablar de la "diversidad sexual", nos estamos refiriendo a esa multiplicidad de identidades de género, expresiones de género y orientaciones sexuales que existen, incluyendo la heterosexual. El término diversidad sexual encierra y celebra 


\section{DERECHO ELECTORAL}

todas las formas de expresión que muestran las diferentes orientaciones sexuales y la identidad de género (CJI, 2013).

\section{Por más derechos para las personas tRanS: CAMINOS Y ALCANCES de LAS REFORMAS EN AMÉRICA LATINA}

Tal como muestra la Tabla 1, el umbral del reconocimiento de derechos en los países de la región que han avanzado en la materia se da entre 2008 y 2012. Es en un período de cuatro años cuando tres países (Argentina, Uruguay y Colombia) inician el camino hacia un mayor número de derechos reconocidos para la población LGBTI, y a partir de ese momento se empiezan a legislar más derechos para la diversidad sexual y de género a nivel nacional de impacto social, civil y político.

\section{Tabla 1}

Reformas políticas a favor de derechos de las personas trans, 2006-2020

\begin{tabular}{|c|c|c|c|c|c|c|c|}
\hline \multirow[b]{2}{*}{ País } & \multicolumn{5}{|c|}{ Derecho reconocido } & \multicolumn{2}{|l|}{ Presidente } \\
\hline & Derecho & $\begin{array}{l}\text { Año en } \\
\text { que se } \\
\text { reconoce }\end{array}$ & Alcance & $\begin{array}{l}\text { Tipo de } \\
\text { derecho }\end{array}$ & $\begin{array}{l}\text { Población } \\
\text { beneficiada }\end{array}$ & $\begin{array}{c}\text { Presidente en } \\
\text { turno } \\
\text { (cuando se } \\
\text { reconoce el } \\
\text { derecho) }\end{array}$ & $\begin{array}{c}\text { Ideología } \\
\text { política } \\
\text { partidaria }\end{array}$ \\
\hline $\begin{array}{l}\text { Argentina } \\
\text { IV }\end{array}$ & $\begin{array}{l}\text { Identidad de } \\
\text { género }\end{array}$ & 2012 & Nacional & $\begin{array}{c}\text { Derecho } \\
\text { identitario }\end{array}$ & $\begin{array}{l}\text { Personas de } \\
\text { la diversidad } \\
\text { de género }\end{array}$ & $\begin{array}{l}\text { Cristina } \\
\text { Kirchner }\end{array}$ & Izquierda \\
\hline $\begin{array}{c}\text { Argentina } \\
\mathrm{V}\end{array}$ & $\begin{array}{c}\text { Ejercicio del } \\
\text { voto (para } \\
\text { personas trans) }\end{array}$ & 2012 & Nacional & $\begin{array}{l}\text { Derecho } \\
\text { político } \\
\text { electoral }\end{array}$ & $\begin{array}{l}\text { Personas de } \\
\text { la diversidad } \\
\text { de género }\end{array}$ & $\begin{array}{l}\text { Cristina } \\
\text { Kirchner }\end{array}$ & Izquierda \\
\hline Bolivia I & $\begin{array}{l}\text { Identidad de } \\
\text { género }\end{array}$ & 2016 & Nacional & $\begin{array}{c}\text { Derecho } \\
\text { identitario }\end{array}$ & $\begin{array}{l}\text { Personas de } \\
\text { la diversidad } \\
\text { de género }\end{array}$ & Evo Morales & Izquierda \\
\hline Brasil X & $\begin{array}{l}\text { Uso de nombre } \\
\text { social para los } \\
\text { contribuyentes }\end{array}$ & 2017 & Nacional & $\begin{array}{l}\text { Derecho } \\
\text { identitario }\end{array}$ & $\begin{array}{l}\text { Personas de } \\
\text { la diversidad } \\
\text { de género }\end{array}$ & Michel Temer & Centro \\
\hline
\end{tabular}




\section{DERECHO EIECTORAL}

\begin{tabular}{|c|c|c|c|c|c|c|c|}
\hline Brasil XI & $\begin{array}{l}\text { Identidad de } \\
\text { género }\end{array}$ & 2018 & Nacional & $\begin{array}{l}\text { Derecho } \\
\text { identitario }\end{array}$ & $\begin{array}{l}\text { Personas de } \\
\text { la diversidad } \\
\text { de género }\end{array}$ & Michel Temer & Centro \\
\hline Chile III & $\begin{array}{l}\text { Identidad de } \\
\text { género }\end{array}$ & 2018 & Nacional & $\begin{array}{l}\text { Derecho } \\
\text { identitario }\end{array}$ & $\begin{array}{l}\text { Personas de } \\
\text { la diversidad } \\
\text { de género }\end{array}$ & $\begin{array}{l}\text { Sebastián } \\
\text { Piñera } \\
\text { Echenique }\end{array}$ & $\begin{array}{l}\text { Centro- } \\
\text { derecha }\end{array}$ \\
\hline $\begin{array}{c}\text { Colombia } \\
\mathrm{V}\end{array}$ & $\begin{array}{l}\text { Identidad de } \\
\text { género }\end{array}$ & 2015 & Nacional & $\begin{array}{l}\text { Derecho } \\
\text { identitario }\end{array}$ & $\begin{array}{l}\text { Personas de } \\
\text { la diversidad } \\
\text { de género }\end{array}$ & $\begin{array}{l}\text { Juan Manuel } \\
\text { Santos }\end{array}$ & $\begin{array}{l}\text { Centro- } \\
\text { derecha }\end{array}$ \\
\hline $\begin{array}{l}\text { Ecuador } \\
\text { III }\end{array}$ & $\begin{array}{c}\text { Ejercicio del } \\
\text { voto (para } \\
\text { personas trans) }\end{array}$ & 2016 & Nacional & $\begin{array}{l}\text { Derecho } \\
\text { político } \\
\text { electoral }\end{array}$ & $\begin{array}{l}\text { Personas de } \\
\text { la diversidad } \\
\text { de género }\end{array}$ & Rafael Correa & Izquierda \\
\hline $\begin{array}{l}\text { Ecuador } \\
\text { IV }\end{array}$ & $\begin{array}{l}\text { Identidad de } \\
\text { género }\end{array}$ & 2016 & Nacional & $\begin{array}{l}\text { Derecho } \\
\text { identitario }\end{array}$ & $\begin{array}{l}\text { Personas de } \\
\text { la diversidad } \\
\text { de género }\end{array}$ & Rafael Correa & Izquierda \\
\hline México I & $\begin{array}{c}\text { Ejercicio del } \\
\text { voto (para } \\
\text { personas trans) }\end{array}$ & 2017 & Nacional & $\begin{array}{l}\text { Derecho } \\
\text { político } \\
\text { electoral }\end{array}$ & $\begin{array}{l}\text { Personas de } \\
\text { la diversidad } \\
\text { de género }\end{array}$ & $\begin{array}{l}\text { Enrique Peña } \\
\text { Nieto }\end{array}$ & $\begin{array}{l}\text { Centro- } \\
\text { derecha }\end{array}$ \\
\hline Panamá I & $\begin{array}{l}\text { Identidad de } \\
\text { género }\end{array}$ & 2006 & Nacional & $\begin{array}{l}\text { Derecho } \\
\text { identitario }\end{array}$ & $\begin{array}{l}\text { Personas de } \\
\text { la diversidad } \\
\text { de género }\end{array}$ & $\begin{array}{c}\text { Martín Torrijos } \\
\text { Espino }\end{array}$ & $\begin{array}{l}\text { Centro- } \\
\text { izquierda }\end{array}$ \\
\hline Perú I & $\begin{array}{l}\text { Identidad de } \\
\text { género }\end{array}$ & 2016 & Nacional & $\begin{array}{l}\text { Derecho } \\
\text { identitario }\end{array}$ & $\begin{array}{l}\text { Personas de } \\
\text { la diversidad } \\
\text { de género }\end{array}$ & $\begin{array}{c}\text { Pedro Pablo } \\
\text { Kuczynski } \\
\text { Godard }\end{array}$ & $\begin{array}{l}\text { Centro- } \\
\text { derecha }\end{array}$ \\
\hline $\begin{array}{l}\text { Uruguay } \\
\text { IV }\end{array}$ & $\begin{array}{l}\text { Identidad de } \\
\text { género }\end{array}$ & 2009 & Nacional & $\begin{array}{l}\text { Derecho } \\
\text { identitario }\end{array}$ & $\begin{array}{l}\text { Personas de } \\
\text { la diversidad } \\
\text { de género }\end{array}$ & $\begin{array}{l}\text { Tabaré Ramón } \\
\text { Vázquez Rosas }\end{array}$ & Izquierda \\
\hline
\end{tabular}

Nota: Observatorio de Reformas Políticas en América Latina UNAM-OEA.

Desde una perspectiva regional, los caminos hacia la aprobación de las reformas y en particular las que protegen los derechos político-electorales de las personas trans han variado según el instrumento institucional utilizado para aprobarlas. Así, la aprobación de reformas en identidad de género se dio a través de promulgación de leyes integrales para la población trans (Uruguay), leyes específicas sobre identidad de género 


\section{DERECHO ELECTORAL}

(Argentina, Chile y Bolivia) ${ }^{13}$, reformas de las leyes y reglamentos del registro civil (Costa Rica, Ecuador, México y Panamá) y reformas a los códigos civiles o familiares (México) (PUICA, 2020b, p. 33).

Varían, también, según el poder del Estado que ha promovido y liderado la reforma. En casi todos los casos, se han aprobado por iniciativa legislativa, aunque en algunos han sido vía decretos del poder ejecutivo, o como resultado de una combinación de participaciones entre los poderes judicial y ejecutivo como sucedió en Colombia, donde una sentencia de la Corte Constitucional fue el antecedente para que se publicara un decreto por parte del poder ejecutivo. Vale resaltar que, en algunos casos, han sido las propias entidades a cargo del registro civil y la identificación las que han adoptado medidas favorables al reconocimiento de la identidad de género (Costa Rica y Panamá) (PUICA, 2020b, p. 33), aunque vale decir que, en el caso de Costa Rica, no se les permite a las personas trans competir en candidaturas a elecciones conforme a su identidad de género.

En 2006, Panamá fue el primer país que dio la posibilidad a las personas trans de rectificar su registro civil; esto por vía administrativa directamente con la entidad. Vale decir; sin embargo, que este artículo de la Ley de Registro Civil ha sido interpretado de manera restrictiva, y solo se ha aplicado en pocos casos de personas con reasignación genital, es decir, el $99 \%$ de las personas trans está quedando excluida de esta posibilidad. Diez años después de haber protegido el derecho a la adopción para parejas de la diversidad sexual, en 2012, Argentina es el primer país en dar un paso crucial en el reconocimiento de derechos de manera sustantiva al aprobar la Ley Nacional de Identidad de Género ${ }^{14}$, y el derecho a sufragar por parte de las personas trans, entre otros, por medio de la cual se permitió la rectificación en los registros públicos del sexo, imagen y nombre de pila con el que fueron inscritos al nacer cuando no coincidían con la identidad de género autopercibida, sin necesidad de verificaciones de terceros.

Uruguay le sigue en 2009 al aprobar la Ley de Identidad de Género ${ }^{15}$; y algunos derechos que fueron parte de reformas posteriores incluyen el derecho a la familia (reproducción asistida) y matrimonio igualitario. Colombia se adelantó con el reconocimiento de derechos como la salud y a

\footnotetext{
13 Ley n. ${ }^{\circ} 807$.

${ }^{14}$ Congreso de la Nación Argentina. Ley n. ${ }^{\circ} 26.743$ de Identidad de Género, artículo 4.

${ }^{15}$ Asamblea General de Uruguay. Ley n. ${ }^{\circ} 19.684$ Integral para Personas Trans.
} 


\section{DERECHO EIECTORAL}

heredar a parejas del mismo sexo, y es en 2015 cuando, junto al derecho a la adopción, aprueba el derecho a la identidad de género ${ }^{16}$. Por su parte, aunque lo hizo extensivo a toda la ciudadanía, Ecuador lo realiza en el año 2016, y aprueba también el ejercicio del voto para las personas trans. Ese mismo año se aprueba una ley en el Perú que permite la rectificación; vale decir, sin embargo, que este procedimiento solo se puede llevar a cabo judicialmente, y es muy difícil que las personas trans puedan ganar esos juicios. Finalmente, el último país de América Latina en hacer ciertas reformas en relación con el tema de la identidad de género de personas trans y el derecho al voto fue México, en particular la Ciudad de México. Posteriormente, y en ausencia de una ley a nivel federal, es el Instituto Nacional Electoral la entidad que, para las elecciones de 2018, decide en la práctica reconocer la identidad de género de los electores a través de un protocolo.

Es decir, lo hace indirectamente atendiendo particularmente el derecho al voto de las personas trans, que se faculta a través del Protocolo para adoptar las medidas tendientes a garantizar a las personas trans el ejercicio del voto en igualdad de condiciones y sin discriminación en todos los tipos de elección y mecanismos de participación ciudadana. Con este instrumento se habilita la posibilidad de que las personas trans puedan votar con plena libertad en las elecciones de julio de 2018. Estas reformas en materia de identidad de género han permitido a miles de personas cambiar su identidad. Según la estimación calculada por el PUICA (2020b p. 31), usando cifras provistas por las autoridades de Argentina, Bolivia, Colombia (Bogotá), Costa Rica, Ecuador, México (diversos estados) y Uruguay, más de 15000 personas en la región han sido reconocidas parcial o integralmente en su identidad de género ${ }^{17}$. Para cerrar, también es importante reconocer otras iniciativas parciales que han tenido lugar en otros países de la región como las de permitir que las personas trans, independientemente de su identidad legal, hicieren la fila para votar con personas de su género de identificación, para evitar la discriminación; esto

\footnotetext{
${ }^{16}$ República de Colombia. Presidente de la República. Decreto 1227/2015 por el cual se adiciona una sección al Decreto número 1069 de 2015, Único Reglamentario del Sector Justicia y del Derecho, relacionada con el trámite para corregir el componente sexo en el Registro del Estado Civil.

${ }_{17}$ A continuación, se incluyen las cifras desagregadas y la indicación del mes y el año en el que se obtuvo el dato. El total incluye 9000 casos en Argentina (febrero, 2020), 270 casos en Bolivia (marzo, 2019), 400 casos en Bogotá, Colombia (septiembre, 2019), 430 casos en Costa Rica (septiembre, 2019), 453 casos en Ecuador (noviembre 2019), aproximadamente 450 casos en Uruguay (noviembre, 2019), aproximadamente 3900 casos en la Ciudad de México (diciembre, 2019), 4 casos en Hidalgo (septiembre, 2019), 162 casos en Michoacán (septiembre, 2019), 20 casos en Nuevo León (noviembre, 2019) y 85 casos en San Luis Potosí (noviembre, 2019).
} 


\section{DERECHO ELECTORAL}

se ha hecho en países donde mujeres y hombres votan en mesas separadas y es, sin duda, un paso hacia una mayor inclusión política de estas poblaciones.

\section{Reformas Político-Electorales para las Personas trans: Agenda Pendiente}

El reconocimiento de la identidad de las personas es uno de los medios que facilita el ejercicio de los derechos a la personalidad jurídica, al nombre, a la nacionalidad, a la inscripción en el registro civil (PUICA, $2020 a$, p. 13) al igual que el derecho a votar en elecciones.

El no reconocimiento de la identidad de género de las personas es equivalente a una situación discriminatoria que es incompatible con los principios democráticos. En efecto, además de todas las facetas de sus derechos, el goce de derechos político-electorales para las personas trans es un imperativo democrático y estas deben poder ejercerlos en plena libertad, sin distinción de ninguna índole y sin temor a ningún tipo de violencia. Acercar a las sociedades latinoamericanas a esta meta es acercarnos a democracias cada vez más consolidadas. Esto importa si miramos la crisis de legitimidad que experimentan las democracias de las Américas, y el impacto que la desigualdad tiene en este descontento; después de todo, tal como indican Pérez y Mainwaring (2008), la desigualdad y la exclusión social se relacionan directamente con el desencanto democrático.

De la revisión de la serie de reformas orientadas a proteger los derechos de las personas LGBTI, y en particular los de las personas trans, se puede establecer una agenda pendiente que debería comprender la institucionalización de las leyes y prácticas democráticas respetuosas de las personas trans, la nivelación de derechos de forma que todos los países, y que tanto el derecho a la identidad como el derecho al voto sean plenamente respetados, y la evolución de las reformas de manera que se pueda incidir en el derecho a las personas trans de ser electas, es decir, la faceta pasiva de los derechos políticos.

En primer lugar, institucionalizar las leyes implica asegurar que todas las reformas puedan tener rango de ley, bien sea por aprobación del Congreso o por decreto; por ejemplo, el avance del derecho a la identidad vía ley o vía protocolo administrativo tiene implicaciones variadas: una ley y un protocolo son dimensiones completamente diferentes con estatus 


\section{DERECHO EIECTORAL}

muy distintos, ya que el protocolo subsana parcialmente y con un impacto mucho menor todo lo que no está legislado, mientras que la ley resuelve de forma más permanente la situación específica sobre la que se legisla o decreta. Es decir, "Ios protocolos sólo son dispositivos que sirven para un momento del tiempo, son de alcance limitado y espacialmente recortados" (Lopez, 2020, p. 20) a diferencia de las leyes que son de carácter más permanente. Estas leyes también deben estar acompañadas de acciones concretas para que, en la práctica, las personas trans puedan acceder a estos derechos tales como programas de educación, campañas de sensibilización, capacitación a operadores electorales, entre otras.

En segundo lugar, las democracias de la región se ven afectadas por las percepciones de desigualdad de la ciudadanía. Todos los países de la región fortalecerán su camino democrático al garantizar el pleno goce de sus derechos, y en especial los político-electorales, a las personas trans. De los 34 países en la región, en la actualidad apenas $29 \%$ o diez países son los que han avanzado con alguna reforma en esta materia. De estos, mientras que nueve se ocuparon de reformas para permitir el reconocimiento de la identidad, apenas tres atendieron deliberadamente el derecho al voto. Es clave poder avanzar en reformas en todos los países de la región para permitir estos dos fundamentales derechos político-electorales para las personas trans.

Finalmente, es preciso empujar la discusión de reformas de tal manera que puedan atender la faceta pasiva de los derechos políticos de las personas LGBTI, y en particular las personas trans: el derecho a ser electas. Hay suficiente evidencia empírica que señala las resistencias de los partidos políticos a promocionar mujeres como candidatas (Dosek et al., 2017), y no es de sorprender que esto se replique en el caso de las personas LGBTI. La experiencia latinoamericana en el diseño e implementación de cuotas y medidas de paridad en la región puede servir de base para un nuevo momento político a nivel regional en donde los partidos políticos incluyan en la oferta al electorado la misma diversidad que existe en nuestras sociedades. 


\section{DERECHO EIECTORAL}

\section{REFERENCIAS BIBLIOGRÁFICAS}

Brown, S. (2002). Con discriminación y represión no hay democracia: the Lesbian and Gay Movement in Argentina. Latin American Perspectives, 29(2), 119-138. https://doi.org/10.1177/0094582X0202900207

Comité Jurídico Interamericano (4 marzo, 2013). Resolución Orientación Sexual, Identidad de Género y Expresión de Género. 82.0 Periodo ordinario de sesiones OEA/Ser.Q 11-15 marzo 2013 CJI/doc.417/12 rev.1 Rio de Janeiro, Brasil, p. 2. Recuperado de http://www.oas.org/es/sla/cji/docs/ cji_agenda_actual_Orientacion_Sexual.pdf

Corrales, J. (2020). The Expansion of LGBT Rights in Latin America and the Backlash. En The Oxford Handbook of Global LGBT and Sexual Diversity Politics. London: Oxford University Press. Recuperado de https://www.oxfordhandbooks.com/view/10.1093/oxfordhb/978019067374 1.001.0001/oxfordhb-9780190673741-e-14?print=pdf

Corrales, J. y Sagarzazu, I. (2019). Not all 'sins' are rejected equally: Resistance to LGBT rights across religions in Colombia. Preparado para la Reunión Annual 2018 de la American Political Science Association, Boston, MA, September 2018.

Corrales, J. y Sagarzazu, I. (2020) LGBTI Rights Expansion in the Global South: Explaining the Diffusion of Same-sex Unions Policy in Latin America. Documento inédito.

Corrales, J. (Dic., 2015). The Politics of LGBT Rights in Latin America and the Caribbean: Research Agendas. Revista Europea de Estudios Latinoamericanos y del Caribe, (100), 53-62.

Corrales, J. (2017). LGBT Rights and Representation in Latin America and the Caribbean: the Influence of Structure, Movements, Institutions, and Culture, LGBT Representation and Rights. Carolina Norte: University of North Carolina, Chapel Hill.

Corrales J. (2020). The Expansion of LGBT Rights in Latin America...and the Backlash. En The Oxford Handbook of Global LGBT and Sexual Diversity Politics. Oxford: Oxford University Press.

Corrales, J. y Pecheny, M. (2010). The comparative politics of sexuality in Latin America. En Javier Corrales y Mario Pecheny (Editores), The Politics of Sexuality in Latin America: A Reader on Lesbian, Gay, Bisexual, and Transgender Rights (pp. 1-30). Pittsburgh PA, United States: University of Pittsburgh Press. 


\section{DERECHO EIECTORAL}

Corte Interamericana de Derechos Humanos (2019). Opinión Consultiva n. ${ }^{\circ} 24$, Identidad de género, e igualdad y no discriminación a Parejas del Mismo Sexo. Recuperado de http://www.corteidh.or.cr/docs/opiniones/ seriea_24_esp.pdf

De la Dehesa, R. (2020). Queering the Public Sphere in Mexico and Brazil: Sexual Rights Movements in Emerging Democracies. Durham, NC: Duke University Press.

Diez, J. (2015) The Politics of Gay Marriage in Latin America: Argentina, Chile and Mexico. Cambridge: Cambridge University Press.

Došek, T., Freidenberg, F., Caminotti, M. y Muñoz, B. (Eds.) (2017). Women, Politics and Democracy in Latin America. New York, Palgrave MacMillan.

Elfenbein, R. (2019) Toward Feminist Socialism? Gender, Sexuality, Popular Power, and the State in Venezuela's Bolivarian Revolution. En Friedman E. \& Tabbush, C. (Editores), Seeking Rights from the Left: Gender, Sexuality and the Latin American Pink Tide (pp 200-234). North Carolina: Duke University Press, Durham, NC.

Encarnación, O. (2016). Out in the periphery: Latin America's gay rights revolution. Oxford: Oxford University Press.

Flores, A. (Ene.-Jun., 2018). El Registro Civil soporte del derecho a la identidad. Las personas trans y el reconocimiento de la identidad de género. Revista de Derecho Electoral, (25), pp. 215-231.

Freidenberg, F. y Muñoz, B. (Editoras) (2016). Reformas políticas a las organizaciones de partidos en América Latina. Lima: Pontificia Universidad Católica de Perú, Organización de los Estados Americanos, Instituto de Investigaciones Jurídicas de la UNAM y Sociedad Argentina de Análisis Político.

Friedman, E. (2012). Constructing 'the same rights with the same names': the impact of spanish norm diffusion Latin American politics and society, 54(4), 29-59.

Friedman, E. (Editor) (2019). Seeking Rights from the Left: Gender, Sexuality and the Latin American Pink Tide. London: Duke University Press.

Garza, R. (2019). Trans: entre lo personal y lo político. Violencias de género y participación político-electoral de las personas trans en México 1990-2016 dentro del sistema electoral mexicano. México: Instituto Nacional Electoral. 


\section{DERECHO EIECTORAL}

Larracoechea, E. (2019). Nicaragua and Ortega's "Second" Revolution: "Restituting the Rights" of Women and Sexual Diversity? En Friedman E. \& Tabbush, C. (Eds.) (2019), Seeking Rights from the Left: Gender, Sexuality and the Latin American Pink Tide (pp. 235-268). Duke University Press, Durham, NC.

López, E. (Oct., 2017). La debilidad del marco legal de las ciudadanías sexuadas frente a las políticas públicas en los escenarios subnacionales, llamados "zonas refugio". Estudios Políticos, Documento de trabajo n. ${ }^{\circ} 24$.

López, E. (14 de febrero., 2019). ¿Qué tan avanzado es el reconocimiento de derechos ciudadanos de las personas LGBT en América Latina? Block Primer Saque. Recuperado de https://oraculus.mx/2019/02/14/que-tan-avanzadoes-el-reconocimiento-de-derechos-ciudadanos-de-las-personas-lgbt-enamerica-latina/

López, E. (2020). Transitar del Protocolo para Garantizar el Voto de las Personas Trans a la Visibilización Legal. Documento inédito.

México. Instituto Nacional Electoral (INE) (2018). Protocolo para adoptar las medidas tendientes a garantizar a las personas trans el ejercicio del voto en igualdad de condiciones y sin discriminación en todos los tipos de elección y mecanismos de participación ciudadana. Recuperado de https://www.ine.mx/wp-content/uploads/2018/06/DECEyEC-ProtocoloTrans.pdf

Munck, G. y Verkuilen, J. (2002). Conceptualizing and Measuring Democracy: Evaluating Alternative Indices. Comparative Political Studies, 35(1), 5-34.

Munck, G. (2005). Measuring Democratic Governance: Central Tasks and Basic Problems. En Deepa Narayan (Editor), Measuring Empowerment: CrossDisciplinary Perspectives (pp. 427-59). Washington, DC: World Bank.

Munck, G. (2009). Measuring Democracy: A Bridge between Scholarship and Politics. Baltimore, Maryland: John Hopkins University Press.

Organización de los Estados Americanos (2013). Convención Interamericana contra toda forma de Discriminación e Intolerancia. Recuperado de http://www.oas.org/es/sla/ddi/tratados_multilaterales_interamericanos_A69_discriminacion_intolerancia.asp

Organización de los Estados Americanos. Programa de Universalización de la Identidad Civil en las Américas (PUICA) (2020a). Lineamientos para la implementación de la opinión consultiva $n .^{\circ} 24$ en el marco del reconocimiento legal de la identidad de género: implicaciones de la 


\section{DERECHO EIECTORAL}

resolución de la Corte Interamericana de Derechos Humanos para las instituciones de registro civil e identificación. Recuperado de http://clarciev.com/identidaddegenero/public/files/SYNERGIA\%20$\% 200 C 24$.pdf.

Organización de los Estados Americanos Programa de Universalización de la Identidad Civil en las Américas (PUICA) (2020b). Panorama del Reconocimiento Legal de la Identidad de Género en las Américas. Disponible en: http://clarciev.com/identidaddegenero/public/files/PANORAMA\%20DEL $\%$ 20RECONOCIMIENTO\%20LEGAL\%20DE\%20LA\%20IDENTIDAD\%20DE\%2 OGENERO\%20EN\%20LAS\%20AMERICAS.pdf

Principios sobre la aplicación de la legislación internacional de derechos humanos en relación con la orientación sexual y la identidad de género (2007). Recuperado de http://yogyakartaprinciples.org/wp-content/uploads /2016/08/principles_sp.pdf

Programa de las Naciones Unidas para el Desarrollo. Secretaría General de la Organización de los Estados Americanos (2004). La democracia en América Latina: Hacia una democracia de ciudadanas y ciudadanos. New York, NY. Recuperado de http://www.bantaba.ehu.es/obs/files/view/ La_democracia_en_Am\%c3\%a9rica_Latina_Parte_1.pdf?revision $\% 5$ fid $=552$ 75\&package $\% 5$ fid $=55247$

Programa de las Naciones Unidas para el Desarrollo. Secretaría General de la Organización de los Estados Americanos (2010). Informe "Nuestra Democracia". México: FCE, PNUD, OEA.

Tabbush, C., Diaz, M., Trebisacce, C y Keller, V. (2019). LGBT Rights Yes, Abortion No: Explaining Uneven Trajectories in Argentina under Kirchnerism (2003-15). En Friedman E. y Tabbush, C. (Editoras), Seeking Rights from the Left: Gender, Sexuality and the Latin American Pink Tide (pp 82-114). Durham, NC: Duke University Press. 